\title{
Optical bullets and double bullet complexes in dissipative systems
}

\author{
J. M. Soto-Crespo \\ Instituto de Óptica, C.S.I.C., Serrano 121, 28006 Madrid, Spain \\ Nail Akhmediev \\ Optical Sciences Group, Research School of Physical Sciences and Engineering, \\ The Australian National University, Canberra ACT 0200, Australia \\ $\mathrm{Ph}$. Grelu \\ Laboratoire de Physique de l'Université de Bourgogne, UMR CNRS 5027, Faculté des Sciences Mirande, Boîte Postale 47870, \\ 21078 Dijon Cedex, France
}

(Received 13 July 2006; published 19 October 2006)

\begin{abstract}
We show that optical light bullets can coexist with double bullet complexes in nonlinear dissipative systems. Coexistence occurs for a relatively large range of the system parameters, and is associated with either marginal stability or bistable existence of the two dissipative soliton species. In the case of marginal stability, spontaneous transformations of single bullets into double bullet complexes are observed. Among the bistable cases, we show how both clockwise and anticlockwise rotating double bullet complexes can be formed out of the phase-controlled interaction of two single bullets. The internal dynamics of pulsating double bullet complexes, with oscillations in both the spatial separation between the two bullets and the bullet shape in time domain is also detailed.
\end{abstract}

DOI: 10.1103/PhysRevE.74.046612

PACS number(s): 42.65.Tg, 47.20.Ky

\section{INTRODUCTION}

A common delusion about dissipative systems is that they can only have losses and thus only decaying solutions. The name "Dissipative system" was coined by Nikolis and Prigogine [1] for systems considered in nonequilibrium thermodynamics. These systems are not isolated, but are kept in contact with an external source that provides energy for the smaller subsystem. Thus dissipation is essential for the transfer of pumped energy to a "cooler." Hence the notion "dissipative system" is more complicated. It assumes that there is also an energy supply part, rather than just losses. Localized structures in these systems, i.e., "dissipative solitons" have their own right to be an established scientific keyword [2].

The term "optical bullet" was invented by Silberberg [3] to depict a complete spatiotemporal soliton, for which confinement in the three spatial dimensions and localization in the temporal domain are achieved by the balance between a focusing nonlinearity and the spreading due to chromatic dispersion and angular diffraction. This occurs while propagation takes place in the medium. The additional balance between gain and loss in dissipative systems provides the shape rigidity of the bullet. In the latter case, they can be called optical dissipative bullets.

Dissipative optical systems admit solitons in one, two, and three dimensions [4]. These formations are stable on propagation, provided the system parameters are chosen in special regions. In addition to stationary solitons, there are pulsating ones which also exist in separate regions of the parameter space. For one- and two-dimensional solitons, the regions of their existence have been studied extensively. The three-dimensional case is still mainly beyond our knowledge.

Dissipative solitons can be considered as elementary building blocks of more complicated structures and patterns in dissipative systems. Thus knowledge of their properties is crucial for understanding the behavior of the system itself. Stable solitons usually have a fixed shape, and the way they are combined into pairs and higher order composite structures is one of the major questions in these studies.

Exact analytical soliton solutions do exist in the case of the one-dimensional cubic-quintic complex GinzburgLandau equation (CCGLE), although all of them have proved to be unstable [5]. Stable solutions of the $(1+1)$-dimensional CCGLE were found only numerically, and it is expected that, in the two- and three-dimensional cases the only hope to find localized structures is by using numerical simulations.

Recently, we have numerically demonstrated the existence of stable light bullets using the three-dimensional CCGLE model in both regimes of chromatic dispersion $[7,8]$. We also studied temporal elongation processes of optical bullets in the normal dispersion regime (transformation into "rockets"). In the present work, we concentrate on the conditions of coexistence of an optical dissipative bullet and a double bullet complex, as well as on the process of the spontaneous transformation of a single bullet into a double bullet complex. A double bullet complex is a localized structure that can be also stationary, pulsating, and rotating. Each behavior is primarily defined by the choice of the parameters of the system and to a lesser extent by the choice of the initial condition. We have found that the stability region of double bullet complexes can overlap with the stability region for single bullets. This causes them to exist simultaneously and creates the possibility of mutual transformations.

An interesting property of double bullet complexes is that their energy can be constant or oscillate with single or double periods. When the total energy of a double bullet complex is constant, it is higher than the sum of the energies of two separate bullets, thus indicating a positive binding energy of the complex. 
The existence of a binding energy in a double bullet complex calls for an analogy with the case of a diatomic molecule. Although there are similarities, there are marked differences too: a stable diatomic molecule of matter can be considered as an isolated system in which the total energy is conserved, and the binding energy should be negative. A double dissipative bullet complex is an open system which requires a continuous supply of energy, and there are no conserved quantities for this system. However, the possible existence of long term stability of these complexes makes the comparison with molecules sustainable, and the analogy can be even quite useful when studying the possible internal pulsations of complexes, as we shall see in Secs. IV and V. Recently, the "optical soliton molecule" terminology appeared explicitly in the titles of published literature and was employed to depict stable aggregates in one-dimensional (1D) or three-dimensional (3D) conservative nonlinear systems $[9,10]$. We should emphasize that in the case of conservative nonlinear systems, there is only marginal stability, so that Hamiltonian "optical molecules" are not stationary and turn to be decaying in the long term. In contrast, our study indicates that the long term stability of a dissipative "optical molecule" relies, when it exists, on the existence of an attractor for the evolution of the dynamical system.

\section{NUMERICAL SIMULATIONS}

Our numerical simulations are based on an extended complex cubic-quintic Ginzburg-Landau equation (CCQGLE) model. This model includes cubic and quintic nonlinearities of dispersive and dissipative types, and we have added transverse operators to take into account spatial diffraction in the paraxial wave approximation. The normalized propagation equation reads

$$
\begin{gathered}
i \psi_{z}+\frac{D}{2} \psi_{t t}+\frac{1}{2} \psi_{x x}+\frac{1}{2} \psi_{y y}+|\psi|^{2} \psi+\nu|\psi|^{4} \psi \\
=i \delta \psi+i \epsilon|\psi|^{2} \psi+i \beta \psi_{t t}+i \mu|\psi|^{4} \psi .
\end{gathered}
$$

The optical envelope $\psi$ is a complex function of four real variables $\psi=\psi(x, y, t, z)$, where $t$ is the retarded time in the frame moving with the pulse, $z$ is the propagation distance, and $x$ and $y$ are the two transverse coordinates. Equation (1) is written in normalized form. The left-hand side contains the conservative terms, viz. $D=+1(-1)$ which is for the anomalous (normal) dispersion propagation regime and $\nu$ which is the saturation coefficient of the Kerr nonlinearity. In the following, the dispersion can have either sign, and the saturation of the Kerr nonlinearity is kept relatively small. The right-hand side includes all dissipative terms: $\delta, \epsilon, \beta$, and $\mu$ are the coefficients for linear loss (if negative), nonlinear gain (if positive), spectral filtering, and saturation of the nonlinear gain (if negative), respectively.

This distributed equation could be applied to the modeling of a wide-aperture laser cavity in the short pulse regime of operation. The model includes the effects of two-dimensional transverse diffraction of the beam, longitudinal dispersion of the pulse, and its evolution along the cavity. Dissipative terms describe the gain and loss of the pulse in the cavity.
Higher-order dissipative terms are responsible for the nonlinear transmission characteristics of the cavity which allows, for example, passive mode-locking. This equation is a natural extension of the one-dimensional complex cubic-quintic Ginzburg-Landau equation (CCQGLE).

We have solved Eq. (1) using a split-step Fourier method. Thus the second-order derivative terms in $x, y$, and $t$ are solved in Fourier space. Consequently, we apply periodic boundary conditions in $x, y$, and $t$. All other linear and nonlinear terms in the equation are solved in real space using a fourth-order Runge-Kutta method. Most of the simulations presented in the paper were done using a numerical grid of 256 points in each of the three dimensions $x, y$, and $t$. We used various values of step sizes along the spatial and temporal domains to check that the results do not depend on the mesh intervals, thus avoiding any numerical artifacts. A typical numerical run presented in this work takes from several hours to several days on a standard modern PC.

In the $(1+1) \mathrm{D}$ case, the cubic-quintic CGLE admits soliton solutions. Moreover, several solutions can exist for the same set of parameters [6]. Not all of them are necessarily stable. The stability is controlled by the parameters of the equation and by the choice of the soliton branch. In this paper we deal with $(3+1) \mathrm{D}$ solitons or optical bullets. We showed in Refs. $[7,8]$ that, in the case of anomalous dispersion as well as for normal dispersion, Eq. (1) admits 3D dissipative solitons, i.e., optical bullets. We show now that this equation also has stable pulsating solutions in both regimes of dispersion. Pulsating solutions turn out to be double bullet complexes, as we have found. The main task is to find a set of parameters where stationary or pulsating solitons exist. In general, we fix five of the parameters, namely $D, \mu$, $\nu, \delta$, and $\beta$, and change $\epsilon$ when looking for stable localized solutions. The initial conditions must be localized when we are looking for localized structures. Their exact shape is relevant but plays a secondary role if only one type of optical bullet exists for a given set of parameters. The shape becomes highly important when several stable solutions coexist. Once a certain kind of localized solution is found for a given set of equation parameters, it can serve as the initial condition for finding solutions at other nearby values of the parameters. By moving slowly in the parameter space, we are able to determine the regions of soliton existence in a relatively easy way.

The natural control parameter of the solution as it evolves is the total energy $Q$, given by the three-dimensional integral of $|\psi|^{2}$ over $x, y$, and $t$ :

$$
Q(z)=\int_{-\infty}^{\infty}|\psi(x, y, t, z)|^{2} d x d y d t .
$$

For a dissipative system, the energy is not conserved but evolves in accordance with the so-called balance equation [11]. If the solution stays localized, the energy evolves but remains finite. Furthermore, when a stationary solution is reached, the energy $Q$ converges to a constant value. When the optical field spreads out, the energy tends to infinity. Another possibility is that the solution dissipates, and then the energy goes to zero. However, if the optical bullet is a 

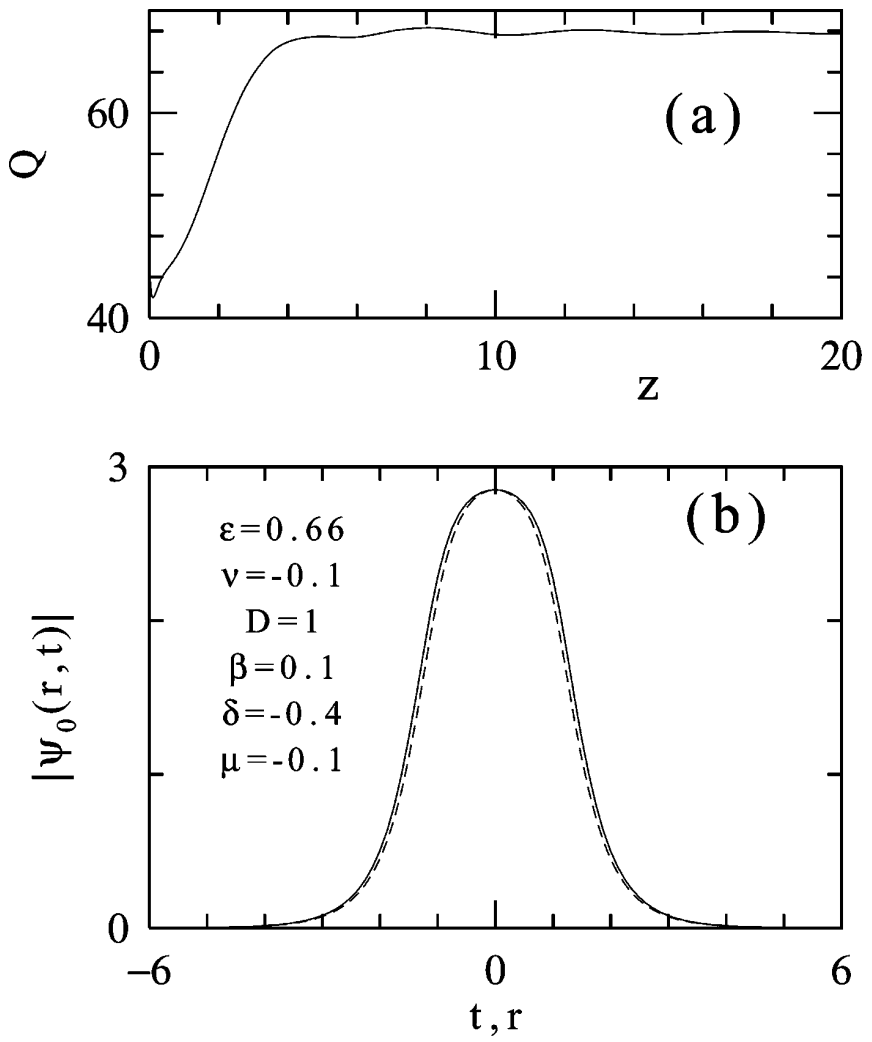

FIG. 1. (a) Total energy $Q$ of the optical bullet defined by Eq. (2) vs propagation distance $z$ for the initial condition given by Eq. (3). The curve shows the convergence of the localized solution towards a stationary optical bullet. (b) Radial (dashed line) $\psi(r, t=0, z \rightarrow \infty)$ and temporal (solid line) $\psi(r=0, t, z \rightarrow \infty)$ profiles of the stable stationary optical bullet. Parameters of the equation for these simulations are shown in (b).

pulsating one, the energy $Q$ is an oscillating function of $z$. We observed all these scenarios in numerical simulations.

\section{STATIONARY OPTICAL BULLETS}

Figure 1(a) shows an example of the evolution of the energy $Q$ along $z$ towards a stationary solution, when we start the simulation with a localized initial condition, such as one with a Gaussian profile in the three dimensions. As our master equation is radially symmetric in space, it seems natural to choose an initial condition that possesses the same symmetry in the $(x, y)$ plane. Specifically, the initial condition that we have chosen is

$$
\psi(x, y, t, 0)=4 \exp \left[-t^{2}-\left(\frac{x}{1.2}\right)^{2}-\left(\frac{y}{1.2}\right)^{2}\right] .
$$

This initial condition has an energy $Q$ that is lower than that for the optical bullet at the chosen set of parameters. Figure 1(a) shows that the energy $Q$ initially increases, but finally converges to a constant value, thus confirming the fact that the localized solution itself tends to converge to a stationary profile.

As expected, the stationary solution is a radially symmetric object in the $(x, y)$-plane. The radial (dashed line) and

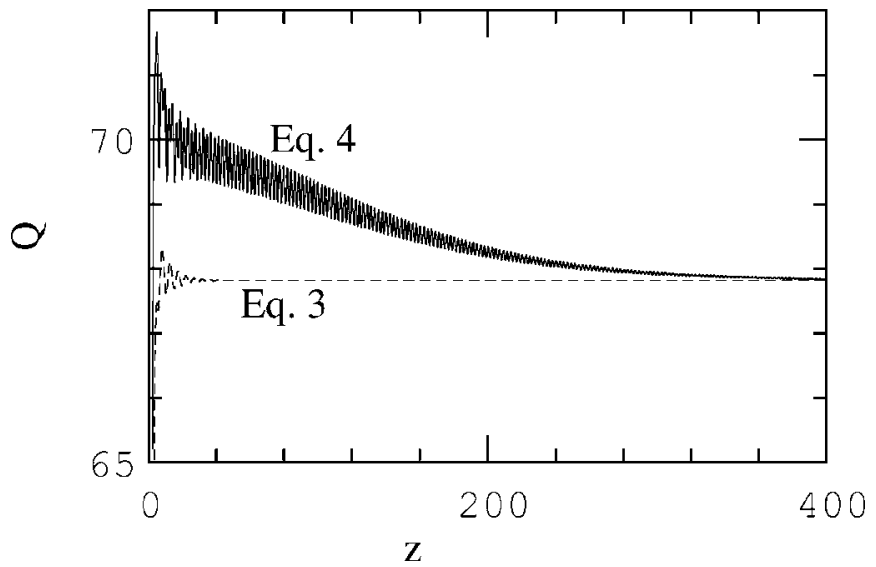

FIG. 2. Energy $Q$ vs propagation distance $z$ when simulations are started from an elliptic localized initial condition (4) (solid line). Dashed line is the same as in Fig. 1(a). A wide range of localized initial conditions, with or without radial symmetry, converge to the same stable bullet.

temporal (solid line) profiles of this stationary solution are shown in Fig. 1(b). These curves represent $\left|\psi_{o}(r, t)\right|$ vs $r=\sqrt{x^{2}+y^{2}}$ at $t=0$ and $\left|\psi_{o}(r, t)\right|$ at $r=0$, respectively. The subscript 0 indicates the asymptotic profile when $z \rightarrow \infty$, i.e., when the stationary solution has been reached. The parameters of the simulation are written inside Fig. 1(b). We can see that, in this case, the temporal profile of the bullet is very similar to, though slightly broader than, that of the radial profile.

Lifting the spatial radial symmetry of the initial condition does not necessarily create a more complicated solution. Solutions without the radial symmetry can be unstable in this range of parameters and the resulting solution is then the one with a symmetric single bullet profile. For example, the initial condition with a strongly elliptic shape

$$
\psi(x, y, t, 0)=4 \exp \left[-t^{2}-\left(\frac{x}{1.1}\right)^{2}-\left(\frac{y}{1.5}\right)^{2}\right]
$$

evolves in a way that reduces ellipticity. Finally it converges to the same radially symmetric optical bullet that was reached when using the initial input given by Eq. (3). This process is shown in Fig. 2. There are decaying oscillations of the elliptical profile of the bullet and corresponding oscillations of the energy before the convergence but the final state, when the oscillations have died out, has the same energy as in Fig. 1(a). For comparison, the dashed line in Fig. 2 shows the evolution when the initial condition is given by Eq. (3) (the same curve as in Fig. 1).

We can notice that convergence from an elliptic initial condition is much slower than for the initial condition (3). This means that stable configurations with elliptic shape may exist for this set of parameters or for a close set of parameters. Thus in order to find a region with optical bullets which lack radial symmetry, we can slightly shift the parameters from the values given in Fig. 1(b). This study is the subject of the following section. 


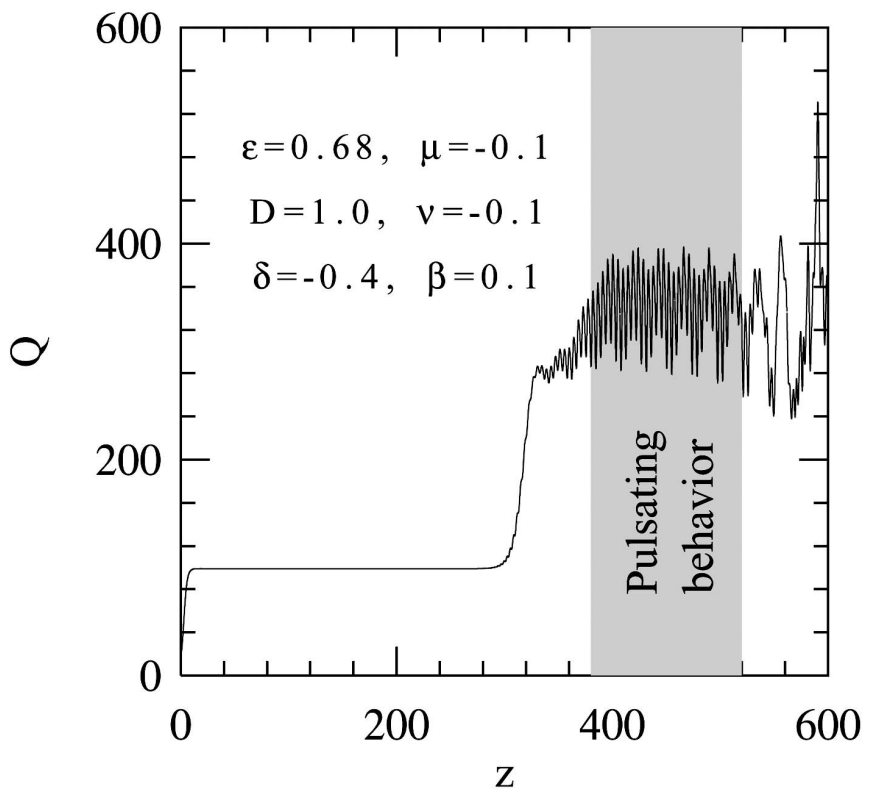

FIG. 3. Energy, $Q$, vs propagation distance $z$ of a localized solution for the set of equation parameters written inside the figure. The initial condition is radially symmetric, thus exciting a stationary radially symmetric bullet (at $10<z<300$ ). This plot also shows the transition (at $z \approx 300$ ) from stationary to pulsating behavior of the optical bullet and a further instability (at $z \approx 520$ ) leading to a chaotic pattern.

\section{PULSATING OPTICAL BULLETS AND DOUBLE BULLET COMPLEXES}

Pulsating optical bullets can be found the same way as stationary solitons. These solutions can be considered as limit cycles and, similarly to the fixed points of a dynamical system, they can be stable or unstable. The solution converges to a pulsating optical bullet if the latter is one of the stable attractors that the dynamical system possesses for a given set of parameters. A pulsating solution can be found with relative ease if it is the only attractor in the phase space. The difficulty arises when stationary and pulsating solutions coexist. Then the choice of the initial conditions becomes crucial.

As we typically used localized initial conditions with spatial radial symmetry, the final result was the stationary one. The pulsating solutions become unveiled as we move to a parameter region where the stationary bullets are unstable. Moving back in the parameter space and keeping the pulsating solution as the initial condition allowed us to reveal the complete branch of pulsating bullets, even if a stationary bullet existed for the same set of parameters. We started the process by finding a set of parameters with marginal stability, where both types of solutions existed for an extended distance of propagation.

Figure 3 shows an example of the $Q(z)$ curve for the evolution of a localized structure when both stationary and pulsating solutions are marginally stable. The initial condition in this case is similar to that given by Eq. (3) and it is not a stationary solution. However, it converges relatively quickly to a stationary bullet, due to the initial radial symmetry of the solution. Then, it remains in a steady state regime that lasts up to $z=300$. It happens that this steady state is unstable relative to perturbations which destroy the radial symmetry in the $(x, y)$ plane. Perturbations in this case are due to the unavoidable accumulation of numerical errors which seeds the instability.

At $z$ around 300, an instability develops and it breaks the spatial radial symmetry of the solution. This instability causes the solution to enter into a pulsating regime. Pulsations continue through the region indicated by a gray stripe in the figure. Pulsations are related to the tendency of the solution to gain an elliptic profile and to split the initial localized state into a double bullet complex. Thus this process is nothing other than the spontaneous transformation of the optical bullet into a bound state of two bullets with an oscillating separation distance between them. Simultaneously, there is a periodic variation of the temporal profile.

The third stage of the evolution in this example after $z$ $\approx 520$ shows the instability of the double bullet complex as well. The number of bullets spontaneously created increases and the structure is further transformed into a complicated pattern that fills the whole numerical grid. The fact that both the steady state and the pulsating solution exist for extended intervals of $z$ reveals the situation when each type of solution is at the margin of stability. Both the stationary bullet and double bullet complex could be stable at nearby points in the parameter space, as we confirm below.

A stable double bullet complex is a pulsating solution of a dynamical system. It is also a limit cycle in an infinitedimensional phase space (see Chap. 1 in [2]). When the parameters of the system are changed, the limit cycle is shifted in the phase space. In numerical simulations, we can use previously obtained pulsating solutions as initial conditions for slightly shifted values of the parameters. When changing $\epsilon$, we allow the optical field to evolve a certain distance before it converges to the new limit cycle. In this way, we can remove any transitory parts of its phase trajectory and be sure that the new pulsating solution (or limit cycle) is stable.

Pulsations of double bullet complexes can be simple harmonic or quasiperiodic. The addition of new frequencies into the motion occurs at certain values of the equation parameters and can be viewed as a bifurcation. In principle, pulsations of a single bullet can keep the spatial radial symmetry or destroy it. In the former case, the bullet changes its size but stays symmetric. We have not observed this type of pulsation for the range of parameters that we used. In the latter case, pulsations are attributed to double bullet complexes before the structure actually splits completely into two separate bullets. In our simulations we observed the opposite process: the merging of two separate bullets into a double bullet complex.

\section{DOUBLE BULLET COMPLEXES WITH CONSTANT ENERGY}

One type of the complexes that we have observed consists of two bullets tightly bound together and rotating around each other. In addition to rotations, the spatial separation between the two bullets can change (i.e., there are vibra- 

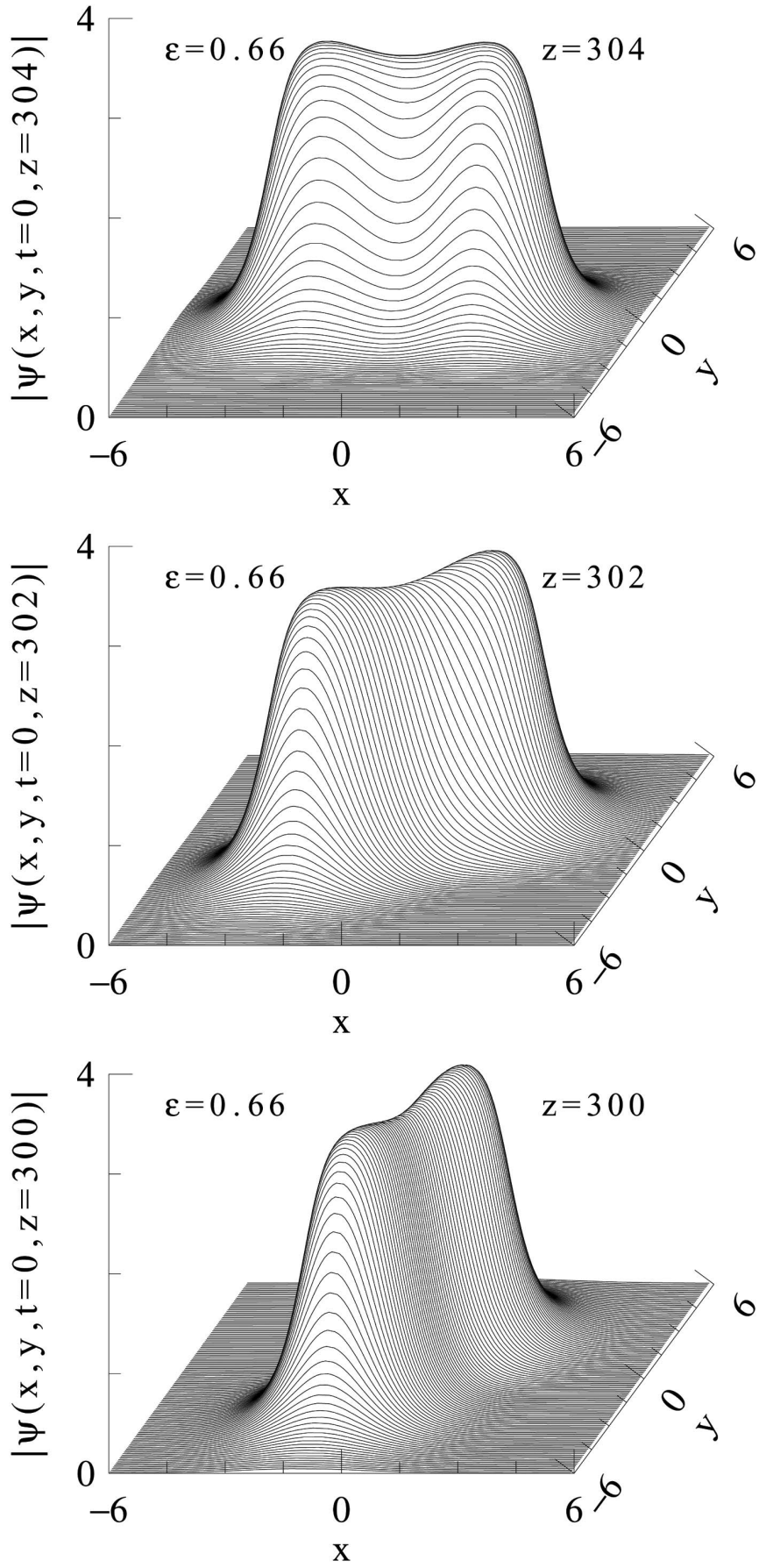

FIG. 4. Spatial profile $\psi(x, y, t=0, z)$ of a rotating double bullet complex. The distance $z$ is shown against each plot. The energy $Q$ for this solution does not depend on $z$.

tions). The latter is followed by oscillating modifications in the temporal profile. The amplitude of these vibrations can vary, depending on the equation parameters. In a limiting case, the amplitude of the vibrations can go to zero, leaving the complex rotating in the plane $(x, y)$, but with a fixed profile both in space and time. The energy $Q$ for these solutions is constant, just as for stationary bullets.

An example of this type of solution is shown in Fig. 4. To excite this double bullet rotating solution, we used, as the initial condition, the pulsating solution that we found for a

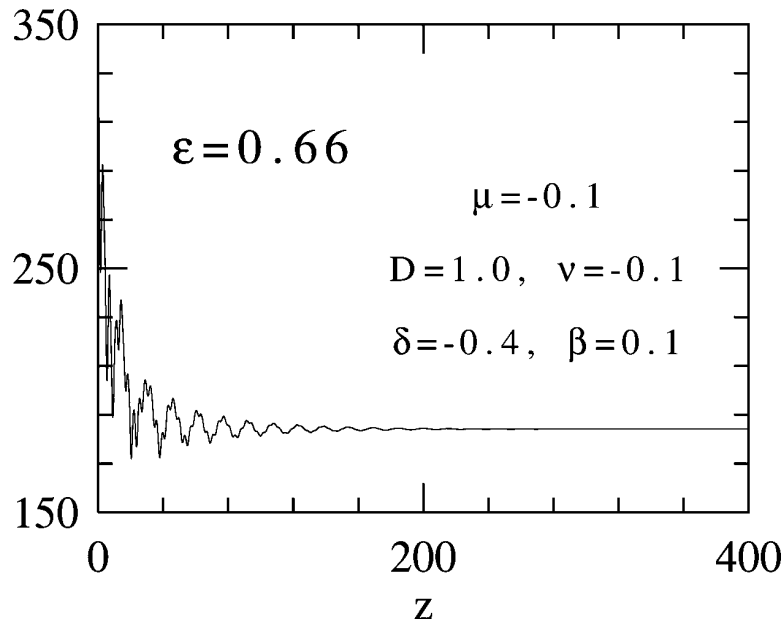

FIG. 5. Energy $Q$ vs propagation distance $z$ for the process of excitation of a double bullet purely rotating complex shown in Fig. 4.

different set of parameters (as the one shown below in Fig. 12). Despite the complicated structure of the initial condition, it converges after a long process to a simple rotating double bullet complex. The energy $Q$ vs $z$ for this conversion is shown in Fig. 5. The final value of the energy (around 184) is more than twice the energy of a single bullet (around 68), thus showing that the two bullets are tightly bound together to form the rotating solution.

The rotation has a fixed angular velocity but can occur in either direction (clockwise or counterclockwise), which is determined by the initial conditions. The distance between the two maxima in the solution does not change in the final stage of the evolution. This solution does exist for the same set of parameters as the single optical bullet. Each formation is stable. Thus they can coexist. A comparison of the spatial profiles of the two solutions in Fig. 6 shows that the complex is indeed a composite state of two stationary optical bullets. The match of the temporal profiles for these two types of solutions (not shown) is almost perfect. The distance between the two bullets in the complex is comparable to the width of each soliton. Hence the amplitude in the center of the complex is comparable to the maximum amplitude of each soliton. That means that the bond between the two bullets is strong, meaning that its energy, $Q$, is well above twice the value of the energy of a single bullet.

In order to check that the double bullet complex can be excited from a variety of initial conditions, we used two stationary bullets located far apart from each other but still interacting through their tails. If their relative phase difference is chosen properly, they do attract and the solution is transformed into a bound state with the resulting curve $Q$ vs $z$ shown in Fig. 7. The two horizontal lines in this figure at $Q \approx 68$ and $Q \approx 184$ mark the fixed energies $Q$ of the single bullet and double bullet complex, respectively. The two oscillating curves correspond to the evolution of two single optical bullets, initially located at the same finite distance, but with different relative phase difference, namely $+10^{\circ}$ and $-10^{\circ}$, respectively.

These two initial conditions converge to a clockwise and a counterclockwise rotating complex, respectively. The start- 


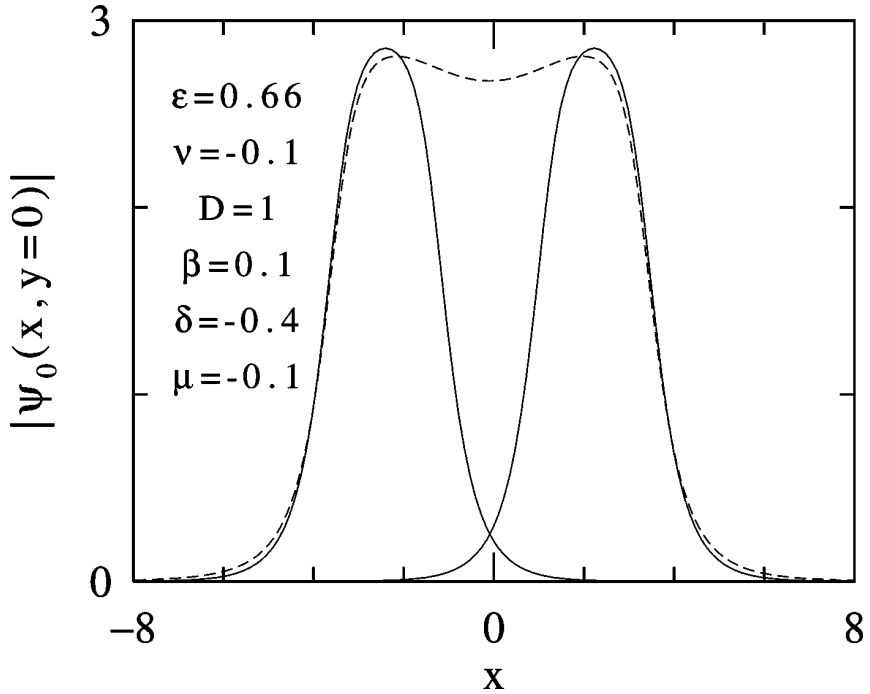

FIG. 6. Profiles $|\psi(x, y=0, t=0, z)|$ of two single optical bullets (solid lines) superimposed on the profile of the rotating double bullet complex (dashed line). Both formations are stable for the same set of equation parameters. Parameters are written inside the figure.

ing energy $Q$ of our initial condition is exactly twice the energy of a single bullet. The resulting energy $Q$ after the collision has taken place and the merging process has finished (around $z=300-400$ ) is clearly the same for the two cases. We can notice that this final energy $Q=184$ is considerably higher than twice the energy of a single bullet. That means that the binding energy for the complex is positive. The bound state of two bullets can be considered as an op-

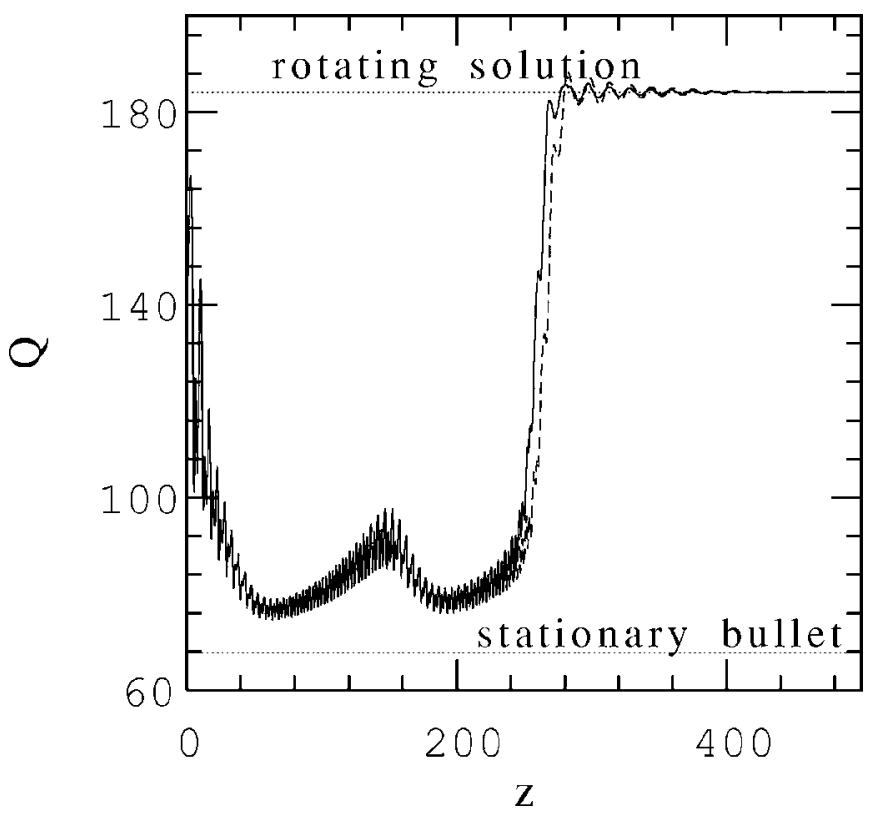

FIG. 7. Energy $Q$ vs $z$ for the process of excitation of a double bullet complex when starting with two well-separated single bullets moving towards each other. The two curves correspond to the evolution of the two single bullets with a relative phase difference of $10^{\circ}$ (solid line) and $-10^{\circ}$ (dashed line). These initial conditions result in a clockwise and a counterclockwise rotating complex, respectively.
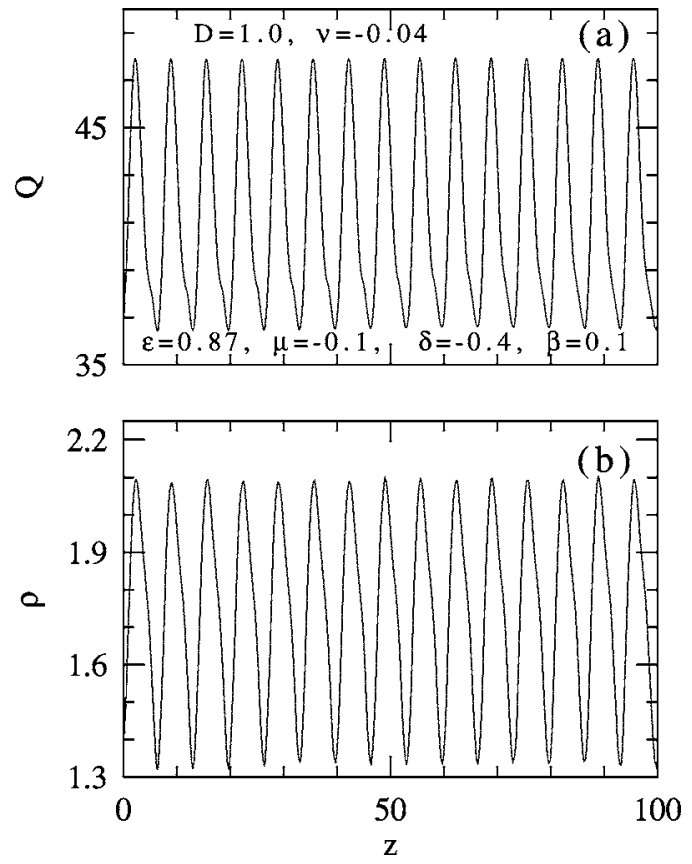

FIG. 8. (a) Energy $Q$ vs $z$ for a double bullet complex with a single period oscillation. (b) The separation between the two maxima in a two bullet complex. This curve is a single periodic function in phase with the curve in (a).

tical molecule, since it possesses long term stability. Moreover, the evolution process described above is analogous to the synthesis of a diatomic molecule out of its two atomic constituents.

\section{DOUBLE BULLET COMPLEXES WITH A SINGLE PERIOD OF OSCILLATIONS}

Let us see, in this section, how the analogy with a molecule can be continued, with the possible existence of vibrating complexes. Indeed, double bullet complexes can be in a ground state with fixed energy or in an excited state with the energy $Q$ oscillating. In particular, we obtained two-bullet complexes with a single period of oscillation between them. The energy $Q$ for these complexes is a simple periodic function of $z$. These oscillations are shown in Fig. 8(a). Oscillations of the energy are caused by the change in the distance between the bullets as the complex evolves in $z$. The relative separation is shown in Fig. 8(b). This curve is also a simple periodic function of $z$. As we can see, the maximal values of $Q$ occur when the separation between the bullets, $\rho$, is also a maximum. This happens just because the size of the complex increases, thus requiring more energy to support it.

Each bullet forming the complex moves in the $(x, y)$-plane along almost a circular trajectory. However, the two bullets rotate around two different points. These points, in turn, rotate slowly around the common "center of mass" making the overall motion complicated. This relative motion is clearly seen in Fig. 9 for $z$ from 0 to 50. The average orientation of the complex in the plane $(x, y)$ is slowly rotating, similar to a precession motion. Vibrations in the spatial domain combine with pulsations in time. The latter have the largest amplitude 


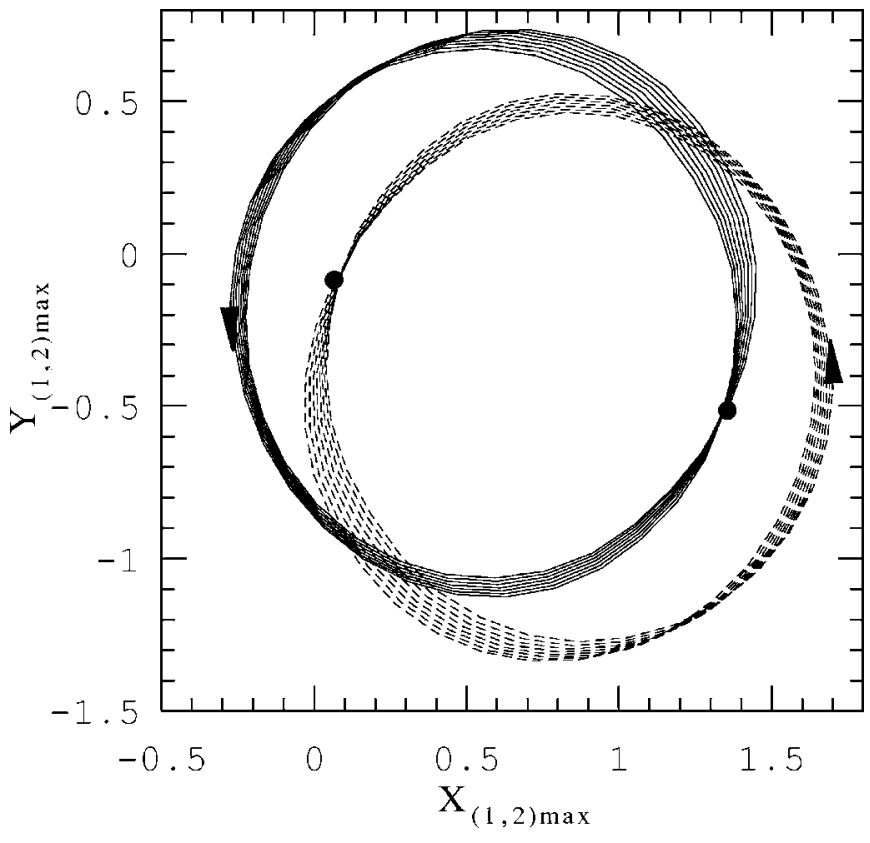

FIG. 9. Trajectories of the maxima of the two bullets that form a stable complex, in the $(x, y)$-plane. The trajectories are shown for half of the distance presented in Figs. 8(a) and 8(b), i.e., for $z$ in the interval $[0,50]$.

at the spatial center of mass of the molecule.

The double bullet complex with oscillating energy can be obtained from the stationary structure by changing the parameters of the system. Thus there is a boundary in the parameter space that separates the two types of structures.

\section{DOUBLE BULLET COMPLEXES WITH TWO OSCILLATION PERIODS}

Oscillations in Fig. 3 observed between $z=400$ and 500 explicitly reveal quasiperiodicity. There are two periods of oscillation rather than one. The only problem is the instability of this periodic solution. To make it stable, we need to change the parameters of the system. Thus, taking as initial condition the pulsating solution for the parameter $\epsilon=0.68$ shown in the gray area in Fig. 3, and reducing $\epsilon$ slightly, we were able to obtain a stable oscillating double bullet complex with two periods of oscillation.

Figure 10 shows $Q$ vs $z$ for a pulsating complex when $\epsilon$ $=0.67$. The rest of the parameters have the same values as the case shown in Fig. 3. Oscillations of the $Q(z)$ curve in Fig. 10 are similar to those in Fig. 3, except that now they last indefinitely along $z$. The two periods involved in this motion are clearly visible. The shorter period is approximately four units in $z$ and the longer period is approximately 20 units. One longer period in Fig. 10 is marked by a solid line. Specifically, it is the part of the curve from $z=80$ to 100 . The plots given below are made for this part of the evolution.

In order to reveal the nature of the oscillations of the optical bullets, we plot separately the evolution of the temporal and spatial profiles. The temporal profile oscillations of the optical field at $(x, y)=(0,0)$ are shown in Fig. 11. The

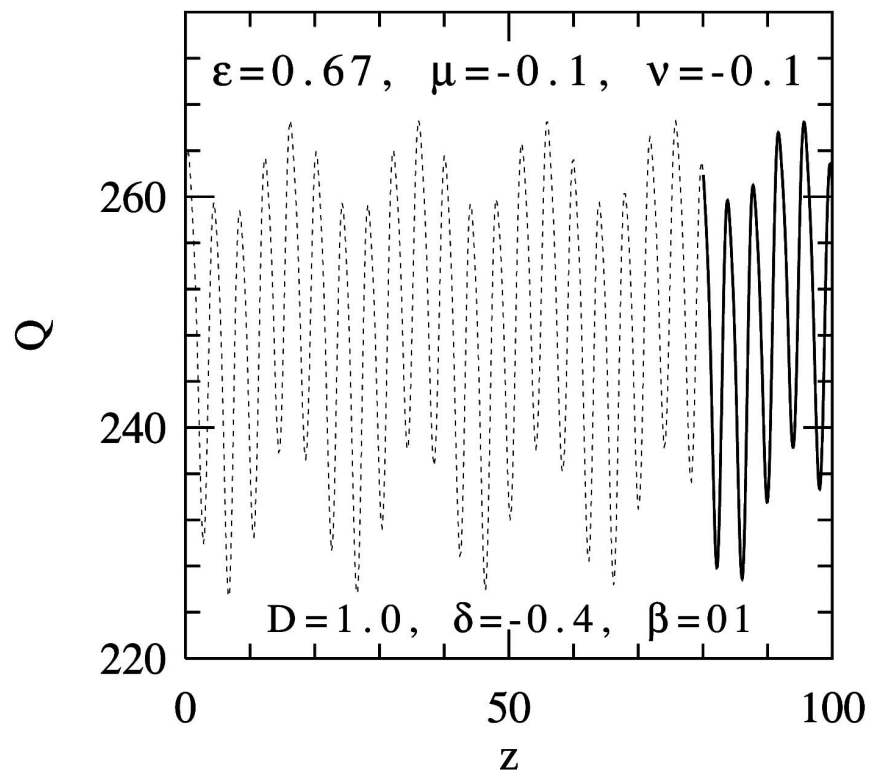

FIG. 10. $Q$ vs $z$ for the double bullet complex with the values of the parameters written inside the figure. The curve reveals double frequency pulsations. The part of the evolution marked by the solid line, from $z=80$ to 100 , is represented in detail in the following three figures.

shape of the bullet changes periodically, but remains symmetric in $t$. The shorter period is clearly visible, while the longer one cannot be appreciated in this figure, although it influences the shape.

Three spatial field profiles at $t=0$ for this simulation are shown in Fig. 12. They correspond to $z=80,82$, and 84, respectively. We can see that the radial symmetry in the $(x, y)$-plane is broken and the solution is a double bullet complex, rather than a single bullet. The complex exhibits an internal dynamics which includes a clockwise rotation around the origin.

In addition to rotation, the separation between the two bullets in the complex oscillates. To see this, we show, in

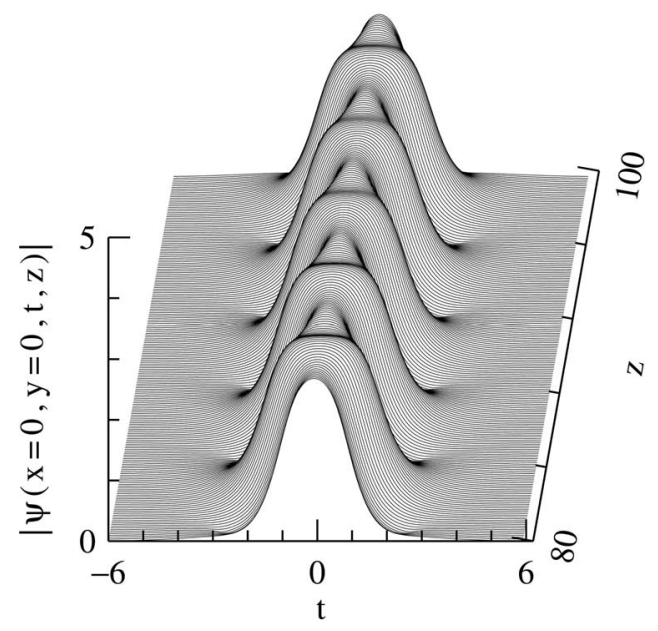

FIG. 11. Evolution of the temporal field amplitude profiles $|\psi(0,0, t, z)|$ at $(x, y)=(0,0)$. The distance $z$ changes from 80 to 100 (continuous line in Fig. 10). 

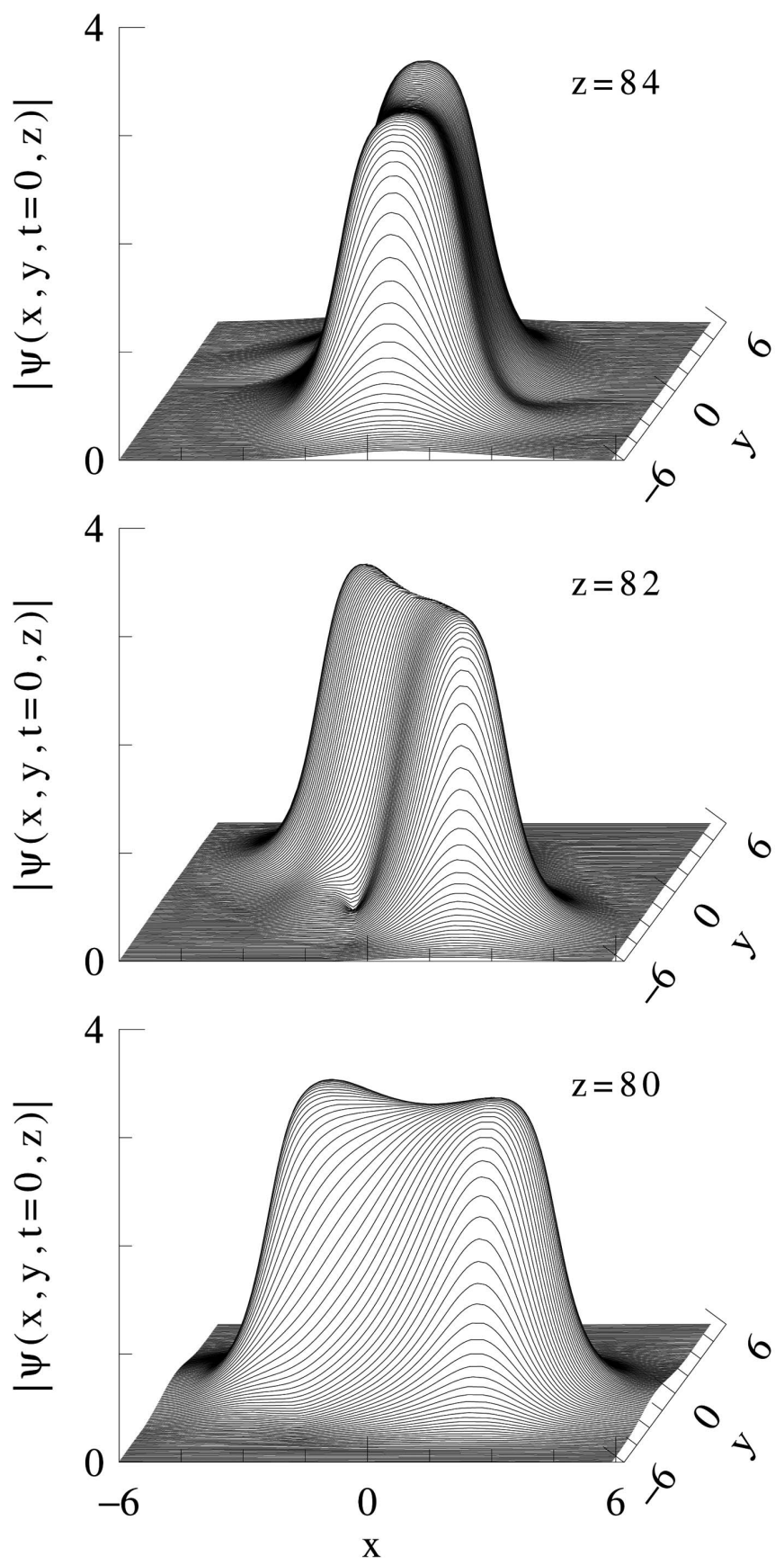

FIG. 12. Three spatial field amplitude profiles $|\psi(x, y, 0, z)|$ at $t=0$ and $z=80,82$, and 84 , respectively.

Fig. 13, the trajectories of the maxima of the optical field. There are two of them on the $(x, y)$ plane, and they occur at $t=0$. They roughly indicate the position of the two bullets in the soliton complex. The trajectories are shown by the dotted and dashed lines, while $z$ changes in the interval $[80,100]$. The initial positions of the maxima at $z=80$ are shown by the two gray dots $(1,2)$. The arrows indicate the direction of the motion, as well as the final position of the maxima at $z$ $=100$. The trajectories look like polygons rather than circles, thus clearly indicating the periodic change in the relative separation between the two maxima. They also indicate that in the three dimensions (defined by the space-time variables

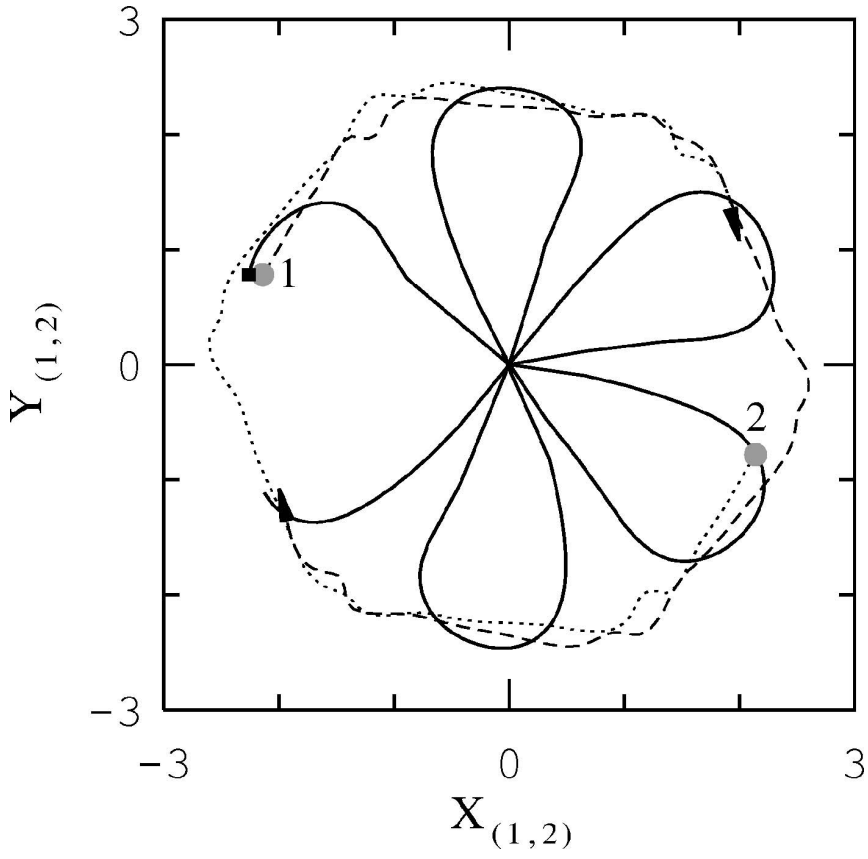

FIG. 13. Trajectories of each of the two maxima (dashed and dotted lines) of the solution shown in Fig. 12 from $z=80$ (big gray dots) to $z=100$ (end of the lines with arrows). The solid line shows the trajectory for one of the maxima of the integrated intensity given by Eq. (5). The starting point is indicated by the black square.

$x, y, t)$, the two bullets always appear to be separated.

On the other hand, experimental measurements could show different behavior. Namely, the quantity that would be detected by a charge coupled device camera with a time response slower than the pulse duration is the field intensity, $|\psi(x, y, t, z)|^{2}$, integrated over time $t$, i.e.,

$$
I(x, y, z)=\int_{-\infty}^{\infty}|\psi(x, y, t, z)|^{2} d t .
$$

The trajectory for one of the two maxima of this quantity is shown in Fig. 13 by the solid line (the trajectory corresponding to the other maximum is similar). This solid line passes through the origin, in contrast to the dashed and dotted lines. When the trajectory passes the origin, the solution must be more elongated in time, thus concentrating the energy closer to the common center of the two bullets.

The two periods of oscillation in the composite solution can be related to a complicated motion inside the complex. One of the motions is the oscillation of the two bullets relative to each other in space, and the other motion involves the pulsations of the shape in the $t$-domain. In a nonlinear problem, these two motions are inseparable and each period can be found in either of the above-mentioned motions. Both periods are revealed in the $Q(z)$ vs $z$ curve of Fig. 10. Double periodic motion of the coupled bullets appears from the single period when we change the equation parameters. The appearance of each new periodic component in the motion can be attributed to a bifurcation. This feature of pulsating solitons in $(3+1) \mathrm{D}$ dissipative systems deserves further study. 


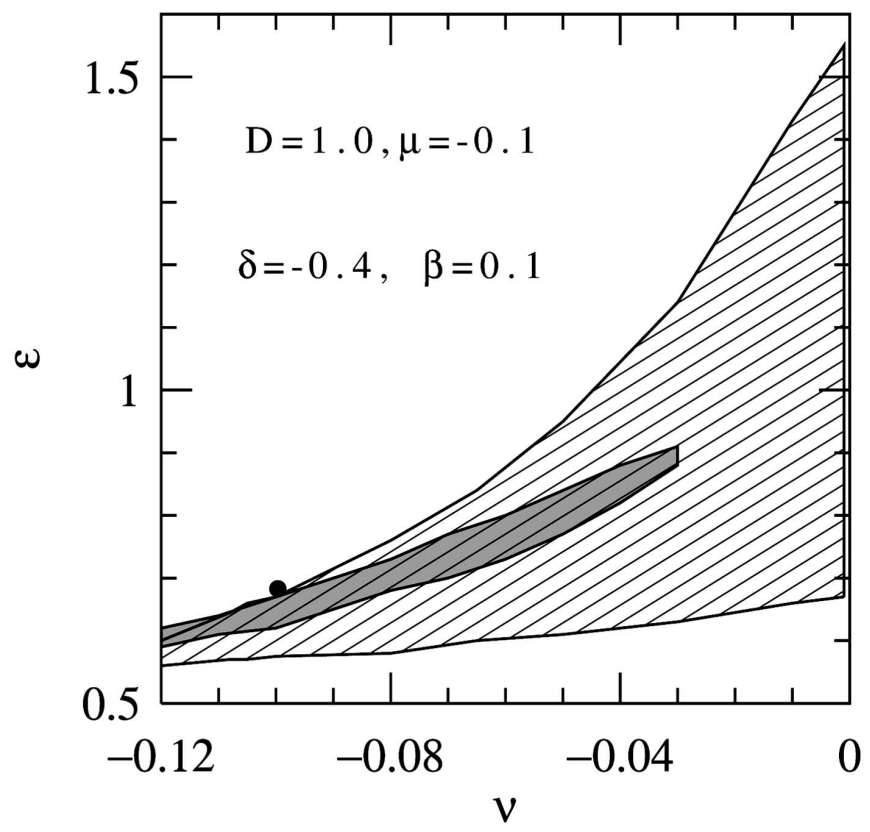

FIG. 14. Region of existence of stable stationary optical bullets in the $(\epsilon, \nu)$-plane (hatched area). The gray area shows the region of existence of stable double bullet complexes. The black dot located at the common edge of the two regions corresponds to the numerical example shown in Fig. 3. Bullets and double bullet complexes are marginally stable at this point.

\section{REGIONS OF EXISTENCE OF OPTICAL BULLETS AND DOUBLE BULLET COMPLEXES}

Optical bullets do exist in finite regions in the parameter space. Each type of double bullet complex also occupies a small finite region. Earlier, in the case of $(1+1) \mathrm{D}$ solitons, we identified at least two independent regions which correspond to qualitatively different dissipative solitons [13]. Namely, we determined two separate regions for stable stationary and pulsating solitons, respectively. The structure of similar regions in the $(3+1) \mathrm{D}$ case can be much more involved. It would be hard to identify all these regions in the whole six-dimensional parameter space. Thus we started with a single point where we were able to find a stable bullet, and extended it step by step to a closed region for a family of these solutions.

To begin with, in the search for optical bullets, we restricted ourselves to varying only two parameters (namely $\nu$ and $\epsilon$ ) while fixing the four others. The results of this search are shown in Fig. 14. In fact, the dashed area in Fig. 14 shows a 2D section of the region in the 6D parameter space where we found stable single optical bullets. The smaller gray region is the one which admits stable double bullet complexes.

The example of optical bullet dynamics presented in Fig. 3 corresponds to the equation parameters at the point shown by the black dot in Fig. 14. This point is located right above the edge of the stability region for both stationary bullets and double bullet complexes. Strictly speaking, both objects are either neutrally stable or weakly unstable at this point. As a result, the localized initial condition cannot lead to any stable structure. Nevertheless, pulsating complexes or stationary bullets may exist for a finite distance of propagation, as we observed in our simulations. Restricting the type of perturbations in the simulations would allow us to excite each type of optical bullet solution for a finite distance $z$. Each type of localized solution becomes stable just below the black point.

Another interesting observation that follows from Fig. 14 is that the region of existence of stable double bullet complexes resides completely inside the region of existence of stationary single bullets. Double bullet complexes in the anomalous dispersion regime mainly coexist with stationary bullets. This is hardly surprising because, to have a stable composite state of two bullets, one would expect that each of them should be stable.

A consequence of this coexistence is that, at certain conditions, there can be transitions from one type of solution to another just as in the case shown in Fig. 3. In particular, if we freeze the radial symmetry of the solution, the double bullet complex may disappear and the stationary single bullet will be excited. In principle, the two regions may overlap only for the set of the parameters that we have chosen. Changing some of the other four parameters of the equation may separate the two regions. However, this study requires tedious work and an enormous amount of numerical simulations.

The two regions in Fig. 14 correspond to the anomalous dispersion regime, as we took $D$ being positive, $D=+1$. We know that dissipative solitons in the $(1+1) \mathrm{D}$ case do exist in the normal dispersion regime as well [12]. In the latter case, the balance between gain and loss plays a major role in the formation of localized structures. We also proved recently the existence of stable $(3+1) \mathrm{D}$ dissipative optical bullets in the normal dispersion regime [8]. Thus, in the present work, we studied regions of soliton existence for $D=-0.1$. Preliminary results are shown in Fig. 15. We keep the same notation for the region with double bullet complexes, as well as for the region with single stationary bullets. This plot seems to be similar to the one presented in Fig. 14. However, the values of the parameters for each region are shifted. In addition to the change of the parameters, we notice that the regions for double bullet complexes and stationary single bullets do not completely overlap. There is a region where only double bullet complexes exist (gray area) as well as a common region of simultaneous existence for two types of solitons. A more detailed study of soliton dynamics in this case will be presented elsewhere.

\section{DISCUSSION}

In this paper, we have studied stationary bullets and various types of double bullet complexes (DBC). An important property that we found is that single bullets and DBCs coexist for a relatively large range of the system parameters that we could explore numerically. This coexistence indicates bistability. At the edge of the above regions when bullets are marginally stable, we can observe spontaneous transformations of single bullets into DBCs after relatively long propagation distances. In addition to regions of bistable coexistence, we have also found regions of parameters where only 


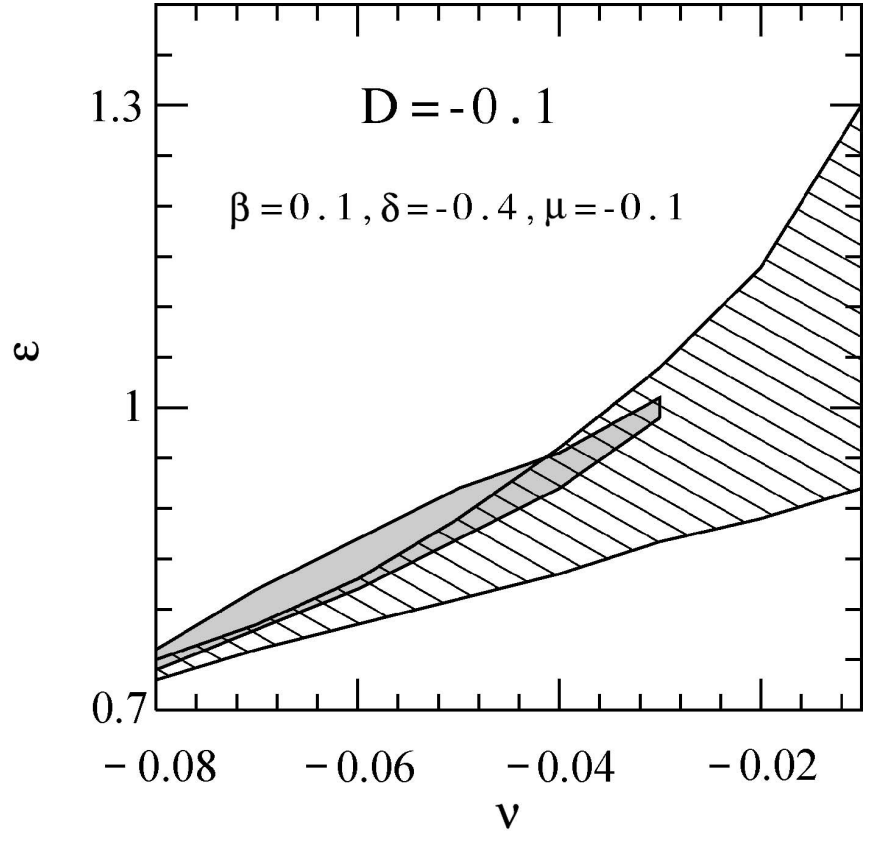

FIG. 15. Region of existence of stable stationary optical bullets in the $(\epsilon, \nu)$-plane (hatched area) for the normal dispersion case $(D=-0.1)$. The gray area shows the region of existence of stable double bullet complexes.

single bullets or double bullet complexes do exist. With these features and the ability to control some of the parameters of the system, we can create a given type of soliton species avoiding any spontaneous transformations.

No doubt, structures more complicated than double bullet complexes can also be found. In particular, we reported earlier on processes of temporal elongation of bullets (transformation into "rockets") and further instabilities that lead to chaotic patterns [8]. Three and more bullets can comprise a variety of complexes that have to be classified in a way similar to the case of DBC. In this sense, our work can be considered to be just a beginning. These preliminary studies show that dissipative systems can serve as a playground for studying the rich dynamics of $(3+1)$-dimensional structures.

Our present study confirms once again the fact that complicated patterns in dissipative systems are composed of elementary building blocks [11]. The latter are essentially solitons. As a simplest example, let us recall the case of pulsating solitons in the $(1+1) \mathrm{D}$ case. Pulsating dissipative solitons [13] have a composite structure. Namely, they consist of two fronts that periodically change the distance between them. Another example of $(1+1) \mathrm{D}$ pulsations is the case of vibrating temporal soliton pairs that are considered recently in [14]. Similarly, pulsating solitons in the $(3+1) D$ case also consist of two simpler objects. They are double bullet complexes. Pulsations are caused by rotations and periodic changes of the distance between the two bullets. Consequently, the $(3+1) \mathrm{D}$ case admits more complicated dynamics, because more degrees of freedom are involved in the problem.

The double bullet complexes that we observed always rotate. Also, the phase difference between the two maxima in each complex is zero. We have not been able to find any complexes with fixed orientation. We know that two in-phase solitons of the nonlinear Schrödinger equation attract each other. Thus it is tempting to explain the stability of the complex by considering that attractive forces compensate for the centrifugal ones. However, the properties of dissipative solitons are different from conservative ones. Even in the $(1+1)$ dimensional case [15], there are several soliton bound states with various separations. Thus the stability of the double bullet complex cannot be easily explained by using a simple particlelike model. Methods similar to those described in Ref. [15] could help in finding stable separation distances between the bullets. However, the rotations result in additional complications in the $(3+1) \mathrm{D}$ case, so that the problem becomes much more involved.

\section{CONCLUSIONS}

In conclusion, based on numerical simulations, we have presented examples of single stable optical bullets and double bullet complexes in dissipative systems modeled by the 3D complex cubic-quintic Ginzburg-Landau equation with an asymmetry between the space and time variables. We showed that the pulsating solutions in this model can be regarded as being formed by two single stationary bullets that rotate around each other. At the same time, the separation between the two bullets oscillates in most of the studied cases, thus creating a vibrational motion which is rather complicated. Three types of these double bullet complexes or "optical molecules" are studied, as well as ways to excite them.

In particular, we showed how both clockwise and anticlockwise rotating double bullet molecules could be formed out of the phase-controlled interaction of two single bullets. The terminology "molecule" is justified by the high stability of complexes, when the parameters of the system are adequately chosen. The dynamics of optical bullets can be controlled by the choice of the system parameters. There is a region of parameters where both a single bullet and a double bullet complex are marginally stable, so that spontaneous transformation between them is possible. We studied, in detail, regions of parameters where coexistence of the two stable dissipative soliton species is possible. Although no complete mapping of the parameter values could be performed for obvious computing reasons, both normal and anomalous regimes of chromatic dispersion are shown to provide regions of coexistence between a stable bullet and a double bullet complex.

These regions of coexistence can provide a fertile ground for future investigations, since the bistability between the optical bullet and the double bullet complex could be used to switch to the desired state using an outside control beam. Also, larger stable "light bullet molecules" comprising more than two bullets are expected from adequately designed interactions between, for instance, a single and a double bullet complex. 


\section{ACKNOWLEDGMENTS}

The work of J.M.S.C. was supported by the M.E.y C. under Contract No. BFM2003-00427 and FIS2006-03376.
N.A. acknowledges support from the Australian Research Council and Ph.G. acknowledges support from Agence Nationale de la Recherche. The authors are grateful to Dr. Ankiewicz for a critical reading of the manuscript.
[1] G. Nicolis and I. Prigogine, Self Organization in Nonequilibrium Systems-From Dissipative Structures to Order Through Fluctuations (Wiley, New York, 1977).

[2] Dissipative Solitons, edited by N. Akhmediev and A. Ankiewicz (Springer, Heidelberg, 2005).

[3] Y. Silberberg, Opt. Lett. 15, 1282 (1990).

[4] N. N. Rosanov, Spatial Hysteresis and Optical Patterns (Springer, Berlin, 2002), Chap. 6.

[5] J. M. Soto-Crespo, N. N. Akhmediev, and V. V. Afanasjev, J. Opt. Soc. Am. B 13, 1439 (1996).

[6] J. M. Soto-Crespo, N. Akhmediev, and K. Chiang, Phys. Lett. A 291, 115 (2001).

[7] Ph. Grelu, J. M. Soto-Crespo, and N. Akhmediev, Opt. Express 13, 9352 (2005).

[8] J. M. Soto-Crespo, Ph. Grelu, and N. Akhmediev, Opt. Express 14, 4013 (2006).
[9] L.-C. Crasovan, Y. V. Kartashov, D. Mihalache, L. Torner, Y. S. Kivshar, and V. M. Perez-Garcia, Phys. Rev. E 67, 046610 (2003).

[10] M. Stratmann, T. Pagel, and F. Mitschke, Phys. Rev. Lett. 95, 143902 (2005).

[11] N. Akhmediev and A. Ankiewicz, Solitons, Nonlinear Pulses and Beams (Chapman \& Hall, London, 1997).

[12] J. M. Soto-Crespo, N. N. Akhmediev, V. V. Afanasjev, and S. Wabnitz, Phys. Rev. E 55, 4783 (1997).

[13] N. Akhmediev, J. M. Soto-Crespo, and G. Town, Phys. Rev. E 63, 056602 (2001).

[14] M. Grapinet and Ph. Grelu, Opt. Lett. 31, 2115 (2006).

[15] D. Turaev, A. Vladimirov, and S. Zelik, Weierstrass Institute fur Angewandte Analysis and Stochastik, Report No. 1152, Berlin, 2006 (unpublished). 